\title{
Regulation of CXCR4 gene expression in breast cancer cells under diverse stress conditions
}

\author{
KLEOPATRA ANDREOU ${ }^{1}$, RAMKUMAR RAJENDRAN ${ }^{1}$, \\ MARIJA KRSTIC-DEMONACOS ${ }^{2}$ and CONSTANTINOS DEMONACOS ${ }^{1}$ \\ ${ }^{1}$ School of Pharmacy and Pharmaceutical Sciences, Stopford Building and ${ }^{2}$ Faculty of Life Sciences, \\ Michael Smith Building, University of Manchester, Oxford Road, Manchester, M13 9PT, UK
}

Received April 6, 2012; Accepted June 8, 2012

DOI: 10.3892/ijo.2012.1643

\begin{abstract}
Chronic inflammation is a critical component in breast cancer progression. Pro-inflammatory mediators along with growth/survival factors within the tumor microenvironment potentiate the expression of pro-inflammatory cytokines (IL-1, IL-6, TNF- $\alpha$ ), chemotactic cytokines and their receptors (CXCR4, CXCL12, CXCL8) and angiogenic factors (VEGF) that often overcome the effect of anti-inflammatory molecules (IL-4, IL-10) thus evading the host's antitumor immunity. Detailed knowledge, therefore, of the regulatory mechanisms determining cytokine levels is essential to understand the pathogenesis of breast cancer. HIF- $1 \alpha$ and NF- $\mathrm{kB}$ transcription factors are important players for the establishment of a pro-inflammatory and potentially oncogenic environment. HIF-1 $\alpha$ is the key mediator of the cellular response to oxygen deprivation and induces the expression of genes involved in survival and angiogenesis within solid hypoxic tumors. The expression of these genes is often modulated by the p53 tumor suppressor protein that induces apoptosis or cell cycle arrest in neoplastic cells. Functional crosstalk between HIF-1 $\alpha$ and p53 pathways mediated by modulators shared between the two transcription factors such as SRC-1 and SIRT-1 differentially regulate the expression of distinct subsets of their target genes under variable stress conditions. In an attempt to shed light on the complex regulatory mechanisms involved in cancer-related inflammation, we investigated the role of the two common p53 and HIF-1 $\alpha$ co-regulators SRC-1 and SIRT-1, in the expression of the highly potent metastatic chemokine receptor CXCR4. Both SRC-1 and SIRT-1 overexpression in DSFX-treated
\end{abstract}

Correspondence to: Dr Constantinos Demonacos, School of Pharmacy and Pharmaceutical Sciences, Stopford Building, University of Manchester, Oxford Road, Manchester, M13 9PT, UK

E-mail: cdemonacos@manchester.ac.uk

Dr Marija Krstic-Demonacos, Faculty of Life Sciences, Michael Smith Building, University of Manchester, Oxford Road, Manchester, M13 9PT, UK

E-mail: m.k.demonacos@manchester.ac.uk

Key words: transcription, inflammation, breast cancer, hypoxia
MCF-7 cells reduced CXCR4 cellular levels implying that both co-regulators are crucial factors in the determination of the metastatic potential of breast cancer cells.

\section{Introduction}

Accumulating evidence supports the notion that most human tumors develop in an environment of chronic inflammatory conditions (1). Consistent with this view several epidemiological studies have connected chronic inflammation with increased risk of cancer development (2). Recent studies have associated DNA damage triggered by inflammation with increased transformation potential of pre-malignant cells within tissues with no indications of underlying infection (3) implying that regardless of the way inflammation is generated its components (inflammatory cells and mediators) ultimately establish an environment that promotes tumor growth and progression.

Hallmarks of cancer-related inflammation include the presence of leukocyte infiltrating cells, cytokines, and peptide mediators of cellular immunity at the sites of tumors (4-6). The balance of pro- (IL-1, IL-6, TNF- $\alpha$ ) and anti-inflammatory cytokines (IL-10, IL-16) and their interdependent interactions within the neoplastic tissue are of pivotal importance in determining the progression and the outcome of the inflammatory reactions (7). TNF- $\alpha$ is a major pro-inflammatory cytokine detected in malignant cells in human breast, ovarian, prostate and bladder tumors as well as in leukemia and lymphomas (8). Since TNF- $\alpha$ is a potent inducer of cancer cell survival and contributes to chronic inflammation and cancer development anti-TNF strategies are used in cancer therapy (9). IL-10 is produced by immune and malignant cells and facilitates cancer cells to escape immune surveillance (10). IL-10 exerts anti-inflammatory effects by inhibiting the production of pro-inflammatory cytokines thus acting as an inhibitor of tumor progression within the tumor microenvironment (7).

A subset of cytokines linked to cancer and in particular to metastasis are the chemotactic cytokines chemokines (11). Deregulation of chemokine networks or inappropriate activation can contribute to inflammatory diseases and malignant transformations $(12,13)$. Signalling via CXCR4 chemokine receptor regulates processes such as cell proliferation, trafficking of immune cells, migration, adhesion and angiogenesis, as part of normal physiology (14). However, since most of these 
events are also required for carcinogenesis, this receptor is the most commonly found in cancer cells (15) and has been shown to play important role in cancer progression as it is involved in the metastatic spread of many human tumors, including breast cancer (13). Its unique ligand, CXCL12, is also expressed within the tumor microenvironment as well as at distant metastatic sites and the CXCR4-CXCL12 axis is essential in the control of invasion and metastasis $(14,16)$.

The cytokine/chemokine network within the tumor microenvironment triggers signalling pathways that induce the expression of genes involved in tumor cell growth and invasiveness, angiogenesis, metastasis and production of more cytokines that affect the existent network and modulate malignant progression. Signal transducer and activator of transcription 3 (STAT3), along with nuclear factor-kappa $\mathrm{B}(\mathrm{NF}-\mathrm{\kappa} \mathrm{B})$ and hypoxia inducible factor 1 alpha $(\mathrm{HIF}-1 \alpha)$ are the main regulatory transcription factors coordinating tumor initiation and progression in tissues where cancer-related inflammation occurs $(5,17)$. Hypoxia is a common feature of inflammatory solid tumors that alters the gene expression profile of genes involved in the regulation of metabolism, angiogenesis, tissue remodelling, proliferation and apoptosis (18). HIF-1 $\alpha$ promotes tumor cell growth and metastasis by upregulating among other genes the expression of CXCR4 in ovarian and breast cancer cells (19). On the other hand, the p53 tumor suppressor mediates both apoptotic cell death and anti-angiogenic effects by repressing HIF-1 $\alpha$ and CXCR4 (20) in hypoxic tumors and mutations inhibiting its pro-apoptotic competence allow inflammation and NF- $\kappa \mathrm{B}$ to exert their tumorpromoting effects (21).

The HIF- $1 \alpha$ and p53 transcription factors interfere with each other's protein stability and transcriptional activity in hypoxic conditions (22-24). Previous work in our laboratory has indicated that the $\mathrm{p} 300 / \mathrm{CBP}$ associated factor (PCAF) is a crucial coordinator of the action of both $\mathrm{p} 53$ and HIF-1 $\alpha$ in hypoxic cancer cells (22). PCAF, p300 and members of the p160 family of the nuclear hormone receptor (NR) co-activators, such as the steroid receptor co-activator 1 (SRC-1), bear intrinsic histone acetyl transferase (HAT) activity thereby modulating directly or indirectly the transcriptional activity of both HIF-1 $\alpha$ and p53 $(22,25,26)$. On the contrary, members of the $\mathrm{NAD}^{+}$-dependent deacetylase (HDAC) family of sirtuins, such as SIRT-1, antagonise HAT activity and target p53 and HIF-1 $\alpha$ for deacetylation in mammalian cells (27). Both SRC-1 and SIRT-1 affect several phenotypic aspects of mammary tumors interfering with the p53 and HIF-1 $\alpha$ pathways $(27,28)$. Herein, we present evidence endorsing the view that SRC-1 and SIRT-1 are involved in the regulation of gene expression of the potent metastatic chemokine receptor CXCR4 in hypoxic breast cancer cells by modulating the transcriptional activity of both p53 and HIF-1 $\alpha$.

\section{Materials and methods}

Cell lines, cell culture and constructs. The human breast carcinoma cell lines MCF-7 (p53 $\left.3^{+/+}\right)$and MDA-MB-231 (p53 $\left.3^{+-}\right)$ (obtained from ECACC) were routinely maintained in Dulbecco's modified Eagle's medium (Sigma-Aldrich, UK), supplemented with $10 \% \mathrm{v} / \mathrm{v}$ foetal bovine serum (Gibco, UK) and $1 \%$ penicillin/ streptomycin $10 \mathrm{U} / \mathrm{ml}$ (Lonza, USA) at $37^{\circ} \mathrm{C}$ in a humidified atmosphere containing $5 \% \mathrm{CO}_{2}$. Cells were treated with $10 \mu \mathrm{M}$ etoposide (Sigma-Aldrich) and $250 \mu \mathrm{M}$ desferrioxamine (DSFX)
(Sigma-Aldrich) for $16 \mathrm{~h}$. Transient transfections were carried out using the Polyfect transfection reagent (Qiagen, Sussex, UK), according to the manufacturer's instructions. Constructs used for exogenous expression included HA-SRC-1 (29) Flag-SIRT-1 (Addgene, Cambridge, MA), pcDNA3 (22), and $\beta$-galactosidase (22). Human CXCR4 luciferase reporter containing 5 consensus HREs was constructed by amplifying the - 1619 to -258 region (counted from the translation initiation site) and inserting it in the pGL3 promoter luciferase vector (Promega, USA).

Quantitative RT-PCR. Quantitative reverse transcription PCR was performed as previously described (22). Briefly, total RNA was extracted from MCF-7 cells using the RNeasy kit (Qiagen), according to the manufacturer's instructions. The RNA was then reverse transcribed to cDNA and used for qPCR analysis using SYBR Green fluorescent probe. Analysis was performed with the Opticon Monitor (Bio-Rad, USA) or Realplex (Eppendorf, UK) software. The primer sequences used for the qRT-PCR reactions are provided in Table I.

Immunoblotting and antibodies. Cells were harvested in high salt lysis buffer $(500 \mathrm{mM} \mathrm{NaCl}, 50 \mathrm{mM}$ Tris- $\mathrm{HCl} \mathrm{pH}$ 7.5, $5 \mathrm{mM}$ EDTA pH 8.0, 0.5\% NP-40, 1\% Triton X-100) containing $1 \mu \mathrm{g} / \mathrm{ml}$ protease inhibitor cocktail (pepstatin, aprotinin, and leupeptin) and $1 \mathrm{mM}$ phenylmethylsulphonyl fluoride (PMSF). Equal amount of protein was loaded on polyacrylamide gels (7.5\%) and resolved by SDS-PAGE. After electroblotting and incubation with primary and secondary antibodies, blots were developed using ECL substrate according to the manufacturer's instructions (Pierce, Thermo Scientific, USA). The antibodies used were anti-HIF-1 $\alpha$ [Calbiochem (H1a67)], anti-p53 [Santa Cruz Biotechnology (sc-126)], anti-CXCR4 [R\&D (MAB 721)], anti-SRC-1 [Abcam (84)], anti- $\beta$-actin [Abcam (8227)], anti-HA [Babco Covance (HA-11)], and anti-Flag [Sigma (M-20)].

Chromatin immunoprecipitation. Chromatin was cross-linked by addition of $1.42 \%$ formaldehyde in the culture medium. Crosslinking was quenched by addition of $125 \mathrm{mM}$ glycine and cells were harvested in IP buffer $(150 \mathrm{mM} \mathrm{NaCl}, 50 \mathrm{mM}$ Tris- $\mathrm{HCl}$ $\mathrm{pH} 7.5,5$ mM EDTA pH 8.0, 0.5\% NP-40, 1\% Triton X-100, $1 \mathrm{mM}$ PMSF, $1 \mu \mathrm{g} / \mathrm{ml}$ protease inhibitor cocktail (pepstatin, aprotinin, and leupeptin), $20 \mathrm{mM} \beta$-glycerol phosphate, and $2 \mathrm{mM}$ sodium orthovanadate). To shear the chromatin, nuclear extracts were sonicated (Bioruptor sonicator), a fraction from each lysate was kept for use as input sample and the rest of the lysate was subjected to immunoprecipitation with $2 \mu \mathrm{g}$ of antibody. The reverse crosslinked DNA fragments were then amplified in PCR reactions with specific primers (Table I) flanking the HREs within the CXCR4 and TNF- $\alpha$ promoter and analyzed on agarose gel electrophoresis.

\section{Results}

CXCR-4, TNF- $\alpha$ and IL-10 mRNA levels in DSFX and etoposide-treated breast cancer cells. Solid tumors bear hypoxic fractions and are often treated with chemotherapeutics such as the topoisomerase II inhibitor etoposide. The therapeutic effect of these compounds depends on their ability to trigger DNA damage and stimulate p53 mediated apoptotic response (30). A previous report from our laboratory along with published 
Table I. Summary of the primers used in ChIP, qRT-PCR and the construction of the CXCR4-luciferase reporter.

\begin{tabular}{lll}
\hline Primer name & \multicolumn{1}{c}{ Sequence $\left(5^{\prime} \rightarrow 3^{\prime}\right)$} & Used for \\
\hline CXCR4 (F) & CATGTGTCTCCCCCTTGAGT & ChIP \\
CXCR4 (R) & TCCGCCTCTAAATTCAGACAA & ChIP \\
TNF $\alpha$ (F) & CTGCCCAAGAAAGAAACCAA & ChIP \\
TNF $\alpha$ (R) & AAAAAGGGAAGGCAAGAAGG & ChIP \\
CXCR4 (F) & CAGCAGGTAGCAAAGTGACG & qRT-PCR \\
CXCR4 (R) & ATAGTCCCCTGAGCCCATTT & qRT-PCR \\
TNF $\alpha$ (F) & AGCCCATGTTGTAGCAAACC & qRT-PCR \\
TNF $\alpha$ (R) & ATGAGGTACAGGCCCTCTGA & qRT-PCR \\
IL10 (F) & TTACCTGGAGGAGGTGATGC & qRT-PCR \\
IL10 (R) & GGCCTTGCTCTTGTTTTCAC & qRT-PCR \\
Rp119 (F) & ATGTATCACAGCCTGTACCTG & qRT-PCR \\
Rp119 (R) & TTCTTGGTCTCTTCCTCCTTG & qRT-PCR \\
CXCR4 (F) LUC & GGTACCGTGCACAAGTGCAGAGAAGG & Construction of the luciferase reporter \\
CXCR4 (R) LUC & GAGCTCAAGAGGGGAGAAGGGAGGAT & Construction of the luciferase reporter \\
\hline
\end{tabular}

A

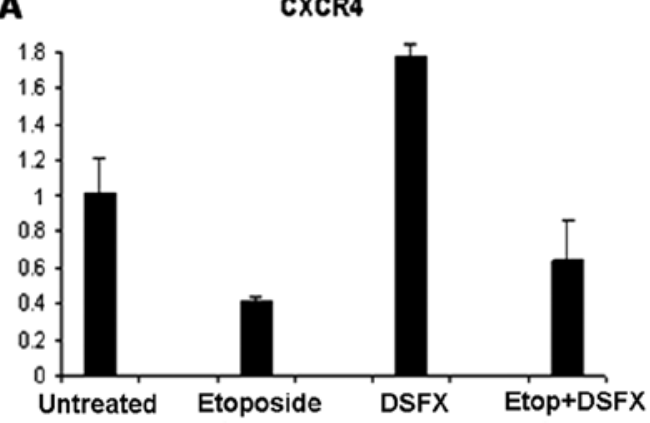

B

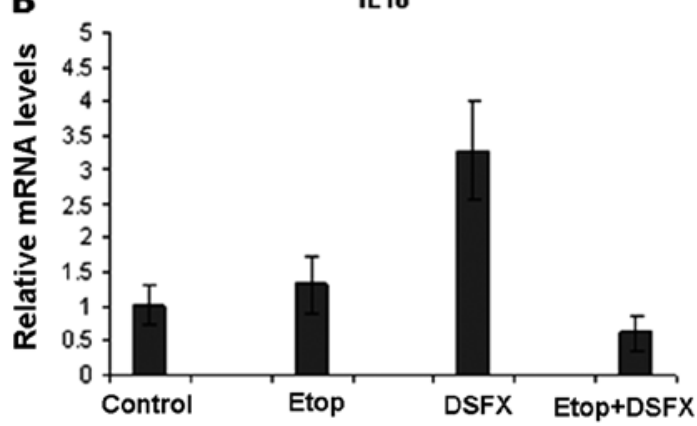

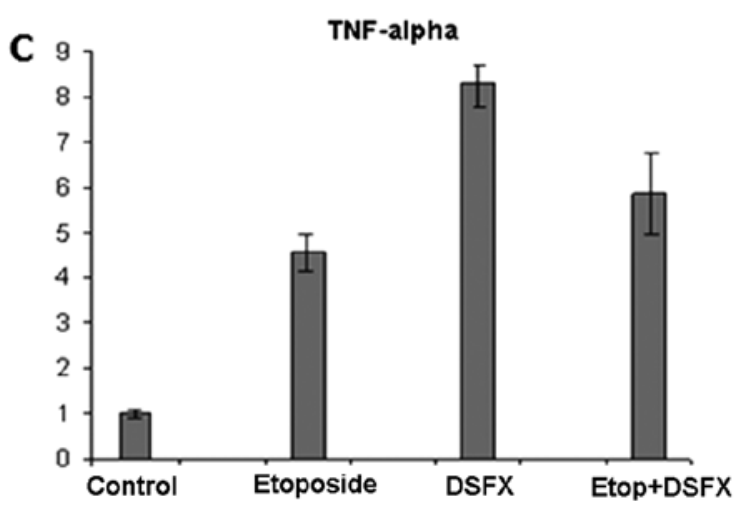

Figure 1. CXCR4 (A), IL-10 (B), TNF- $\alpha$ (C) mRNA levels in DSFX, etoposide and combination of etoposide and DSFX-treated MCF-7 cells. Graphs show mRNA levels measured in untreated, etoposide, DSFX and both etoposide and DSFX-treated cells normalized to Rpl19. The relative gene/Rpl19 mRNA level in the untreated cells was arbitrarily set to 1 . Values represent the mean of three (A), two (B) and one (C) independent experiments (95\% CI) in which each point was performed in duplicate.

observations from other groups, indicate that the function of $\mathrm{p} 53$ differs significantly in hypoxic compared to normoxic environment (22). Taken these observations into account, the expression pattern of $C X C R 4$, which is a gene targeted by both HIF-1 $\alpha$ (19) and p53 (20), was followed in MCF-7 cells under conditions where both $\mathrm{p} 53$ and HIF-1 $\alpha$ were transcriptionally active in order to delineate the molecular mechanisms involved in the regula- tion of its gene expression. Reduced $C X C R 4$ mRNA levels by $50 \%$ were observed in etoposide-treated compared to untreated MCF-7 cells (Fig. 1A). In agreement with earlier reports (19) increased by 1.7 -fold $C X C R 4$ gene expression was identified in MCF-7 cells treated with the hypoxia mimicking agent DSFX compared to untreated cells (Fig. 1A). Reduced CXCR4 mRNA levels were observed in MCF-7 cells treated with combination 
A

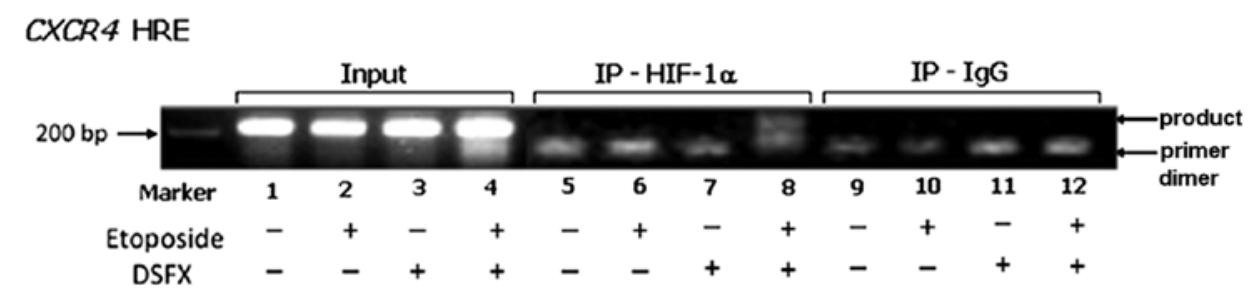

B

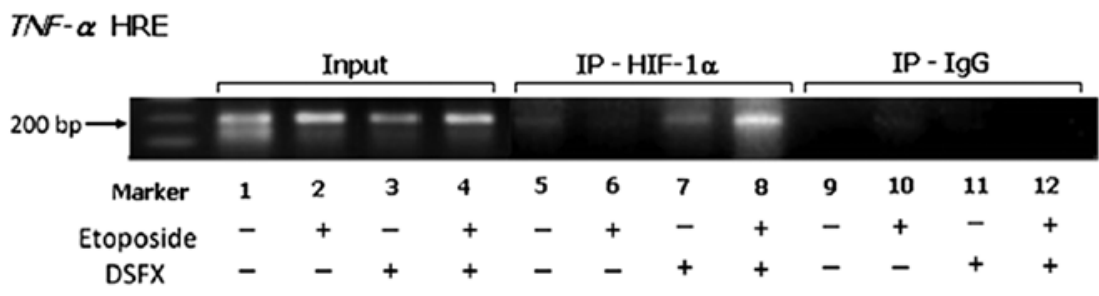

Figure 2. Chromatin immuno-complexes from untreated, etoposide, DSFX, or etoposide and DSFX-treated MCF-7 cells were precipitated using a specific HIF-1 $\alpha$ (lanes 5-8) or an irrelevant non-specific antibody (lanes 9-12) as negative control. The precipitated DNA was amplified with specific primers flanking the regions containing the HREs present in $C X C R 4(\mathrm{~A})$ and $T N F-\alpha(\mathrm{B})$ promoter.

of etoposide and DSFX compared to untreated cells (Fig. 1A), but these levels were 1.7-fold higher than those measured in cells treated with etoposide alone (Fig. 1A). Given that MCF-7 cells bear wild-type p53, the reduced CXCR4 levels observed in cells subjected to the combined treatment compared to those in DSFX-treated cells implied a dominant p53 suppressive action over HIF-1 $\alpha$ (Fig. 1A).

Putative hypoxia responsive elements (HREs) were identified within the regulatory regions of IL-10 and TNF- $\alpha$ promoters (data not shown) and their mRNA levels were monitored in a similar manner in MCF-7 cells. Induction of IL-10 and TNF- $\alpha$ gene expression was detected in DSFX-treated cells (3-and 8-fold respectively) compared to that in non-treated cells (Fig. 1B and C respectively). Combination of etoposide and DSFX treatment repressed $I L-10$ expression by $\sim 40 \%$ but induced $T N F-\alpha$ mRNA levels by 5 -fold compared to untreated cells (Fig. 1B and C). Notably, etoposide treatment counteracted the stimulatory effect of DSFX treatment in the case of both IL-10 and TNF- $\alpha$.

$H I F-1 \alpha$ recruitment to the regulatory regions of the promoter of $C X C R 4$ and $T N F-\alpha$. Given that etoposide-treated MCF-7 cells in hypoxia mimicking conditions exhibited lower $C X C R 4$ mRNA levels compared to those observed in cells treated with DSFX only (Fig. 1A) and taking into account the fact that CXCR4 is known HIF-1 $\alpha$ transcription target (19) we were interested in investigating the mechanisms coordinating the recruitment of $\mathrm{HIF}-1 \alpha$ to the $C X C R 4$ promoter in MCF-7 cells under diverse stress conditions. MCF-7 cells were treated with etoposide, DSFX or combination of both and the HIF-1 $\alpha$ recruitment to the CXCR4 promoter was investigated employing chromatin immunoprecipitation (ChIP) assays. It is worth noting that the HRE followed in this study was different than that assessed by Schioppa et al (19). Interestingly, we observed that HIF-1 $\alpha$ was recruited to the $C X C R 4$ promoter only in MCF-7 cells treated with both DSFX and etoposide (Fig. 2A, lane 8) whereas that was not the case in MCF-7 cells treated with DSFX alone (Fig. $2 \mathrm{~A}$, lane 7). These findings suggested that the HIF-1 $\alpha$ transcrip- tional complex recruited to the $C X C R 4$ promoter in DSFX and etoposide-treated MCF-7 cells differed from that present in these cells treated only with DSFX.

In similar manner ChIP experiments were carried out in MCF-7 cells treated with etoposide, DSFX or combination of both and the HIF- $1 \alpha$ recruitment to the putative HRE identified 6923-bp upstream of the translation start site of the TNF- $\alpha$ promoter was assessed. HIF-1 $\alpha$ was recruited to the $T N F-\alpha$ promoter in DSFX-treated MCF-7 cells (Fig. 2B, lane 7) providing evidence that $T N F-\alpha$ induction in hypoxia mimicking conditions (Fig. 1C) was a result of direct HIF-1 $\alpha$ mediated trans-activation. Moreover, HIF-1 $\alpha$ was recruited to the $T N F-\alpha$ promoter in MCF-7 cells treated with combination of DSFX and etoposide (Fig. 2B, lane 8). Taken together these results implied the existence of a selective mechanism directing the recruitment of HIF-1 $\alpha$ in specific subsets of its transcriptional targets under diverse stress conditions.

Protein levels of CXCR4 in breast cancer cells. Western blot analysis was performed to follow the CXCR4 protein levels under conditions of DNA-damage, hypoxia mimicking and combination of the two stresses. Two breast cancer cell lines differing in the 533 status (MCF-7 and MDA-MB-231) were used for this analysis. Elevated CXCR4 protein levels were observed under these stresses in wild-type p53 MCF-7 cells, compared to non-treated cells (Fig. 3A, compare CXCR4 lanes 2-4 to lane 1). Combination of DSFX and etoposide treatment resulted in higher CXCR4 protein levels compared to those observed in cells treated with either etoposide or DSFX individually (Fig. 3A compare lane 4 with lanes 2 and 3 respectively). The same analysis was performed in MDA-MB-231 cells expressing high levels of mutated p53G280A (31). Elevated CXCR4 protein levels were observed in etoposide and DSFX-treated MDA-MB231 cells (Fig. 3B compare lanes 2 and 3 with lane 1 respectively) whereas combined etoposide and DSFX treatment of these cells resulted in downregulation of the CXCR4 protein levels (Fig. 3B compare lane 4 with lane 1). 


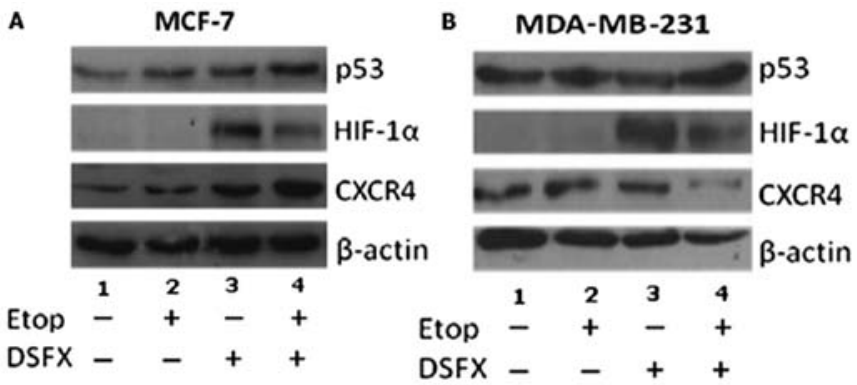

Figure 3. CXCR4 protein levels in etoposide and DSFX-treated breast cancer cells. Cellular extracts from etoposide, DSFX or both etoposide and DSFXtreated MCF-7 (A) or MDA-MB-231 (B) cells were subjected to PAGE analysis. Results are representative of three independent experiments.

SRC-1 and SIRT-1 affect CXCR4 gene expression in hypoxia mimicking conditions. SRC-1 is a HIF-1 transcriptional coactivator in normoxic and hypoxic cells $(26,32)$ whereas SIRT-1 deacetylase, exerts SRC-1 opposing effects in the androgen receptor signalling pathway in prostate cancer cells (33) and has been linked to hypoxia-mediated transcription (34). Given the functional and physical interplay of SRC-1 and SIRT-1 with HIF-1 $\alpha(26,32,35)$ together with the identification of multiple putative HREs in the regulatory region of the CXCR4 promoter triggered our interest to investigate the effect of these two factors on the activity of $C X C R 4$ promoter in hypoxic breast cancer cells. To assess the role of SRC-1 and SIRT-1 on CXCR4 promoter activity under hypoxia mimicking conditions, we performed luciferase reporter assays in MCF-7 cells transfected with the CXCR4-luc reporter and exogenously expressing either SRC-1 or SIRT-1 under normoxic or hypoxia mimicking conditions.

Initial reduction of $C X C R 4$ luciferase reporter activity was evident in MCF-7 normoxic cells expressing exogenously transfected SRC-1 (Fig. 4A compare bar 1 with bar 2) which gradually increased following increasing amounts of transfected SRC-1 (Fig. 4A compare bars 3, 4 with bar 2). CXCR4 luciferase reporter activity was lower in cells treated with the hypoxia mimicking agent DSFX compared to the untreated cells
(Fig. 4A compare bars 1-4 with bars 5-8). Slight induction of the CXCR4 luciferase reporter was observed in cells transfected with the highest amount of SRC-1 (Fig. 4A compare bars 6 and 7 to bar 8) suggesting that overexpressed SRC-1 can reverse the suppressive effect of hypoxia on the activity of the CXCR4 luciferase reporter.

In contrast to SRC-1, exogenous expression of SIRT-1 gradually reduced the $C X C R 4$ luciferase reporter activity in a dose-dependent manner in both normoxic (Fig. 4B, compare bars 2-4 to bar 1) and hypoxia mimicking conditions (Fig. 4B, compare bars 6-8 to bar 5) in MCF-7 cells lending support to the view that SIRT-1 exacerbates the repressive effect of hypoxia on the activity of the CXCR4 luciferase reporter.

Effect of SRC-1 and SIRT-1 on CXCR4 protein levels. To explore further the effect of SRC-1 and SIRT-1 in the modulation of the CXCR4 expression, we assessed the CXCR4 protein levels in untreated, etoposide and DSFX-treated MCF-7 cells exogenously expressing either SRC-1 (Fig. 5A and B) or SIRT-1 (Fig. 5C and D). CXCR4 protein level in each condition was compared to the level of this protein in cells transfected with the empty vector pcDNA3. SRC-1 transfection resulted in an average $50 \%$ increase of CXCR4 protein in untreated normoxic cells (Fig. 5A and B, compare black bar 1 to grey bar 1). Etoposide treatment increased the CXCR4 protein levels by $40 \%$ in SRC-1 exogenously expressing cells compared to pcDNA3-transfected cells (Fig. 5A and B, compare black bar 2 to grey bar 2). In contrast, $29 \%$ decrease of CXCR4 protein levels were observed in DSFX-treated MCF-7 cells overexpressing SRC-1 compared to non-transfected cells (Fig. 5A and B, compare black bar 3 with grey bar 3). Combination of etoposide and DSFX treatment in SRC-1 overexpressing cells reduced CXCR4 protein levels by $16 \%$ compared to cells transfected with pcDNA3 under the same conditions (Fig. 5A and B compare black bar 4 with grey bar 4).

SIRT-1 transfection increased CXCR4 protein levels in untreated cells but reduced the protein levels of this receptor in cells treated with etoposide, DSFX and combination of both compared to untransfected cells (Fig. 5C and D). SIRT-1 trans-
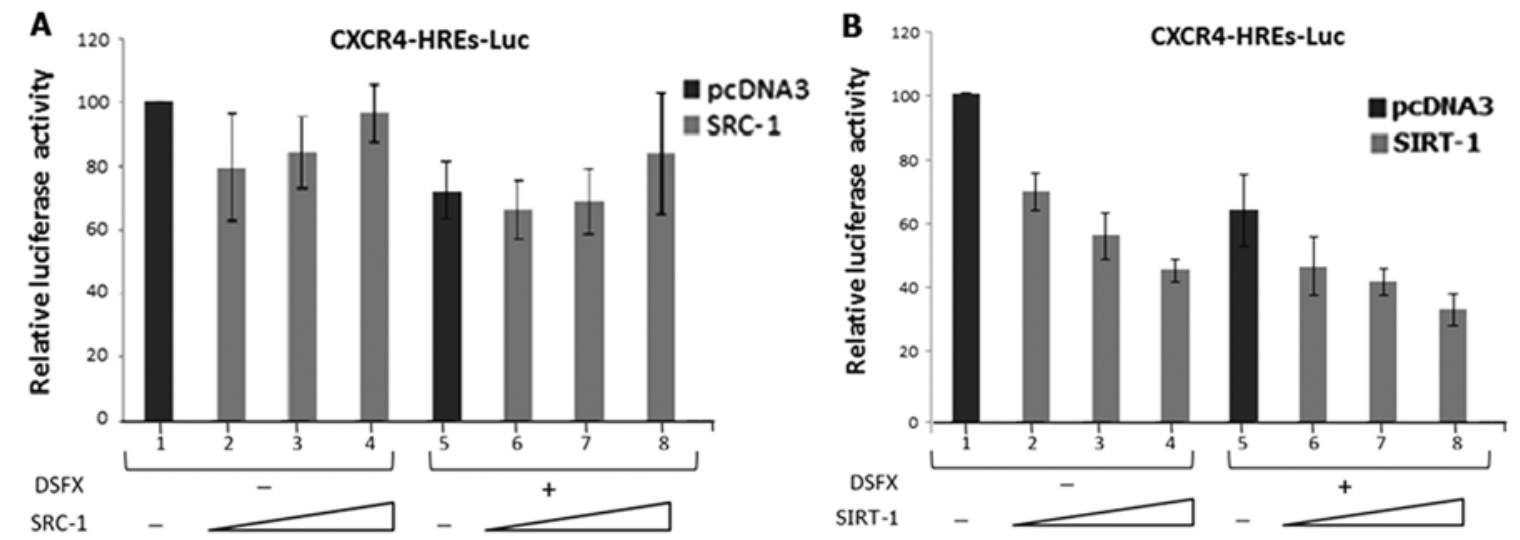

Figure 4. Effect of SRC-1 (A) and SIRT-1 (B) on CXCR4 luciferase reporter activity in untreated or DSFX-treated MCF-7 cells. Values represent the means of three independent experiments. The relative luciferase activity of the CXCR4-luc reporter in untreated cells transfected with pcDNA3 was arbitrarily set to 100 after normalization to $\beta$-galactosidase activity.

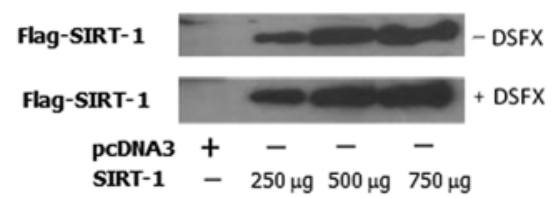


A

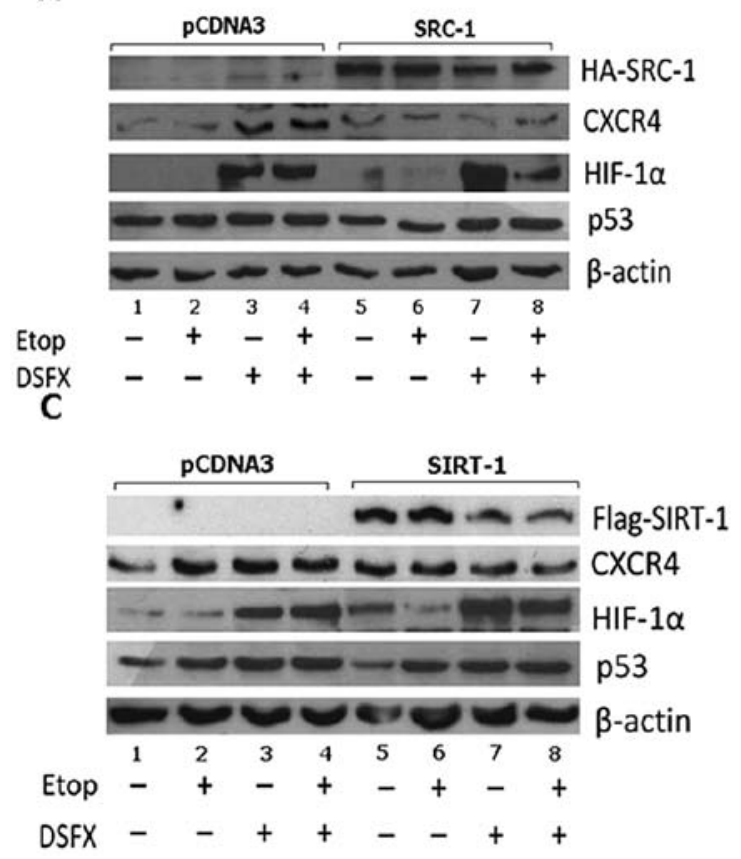

B
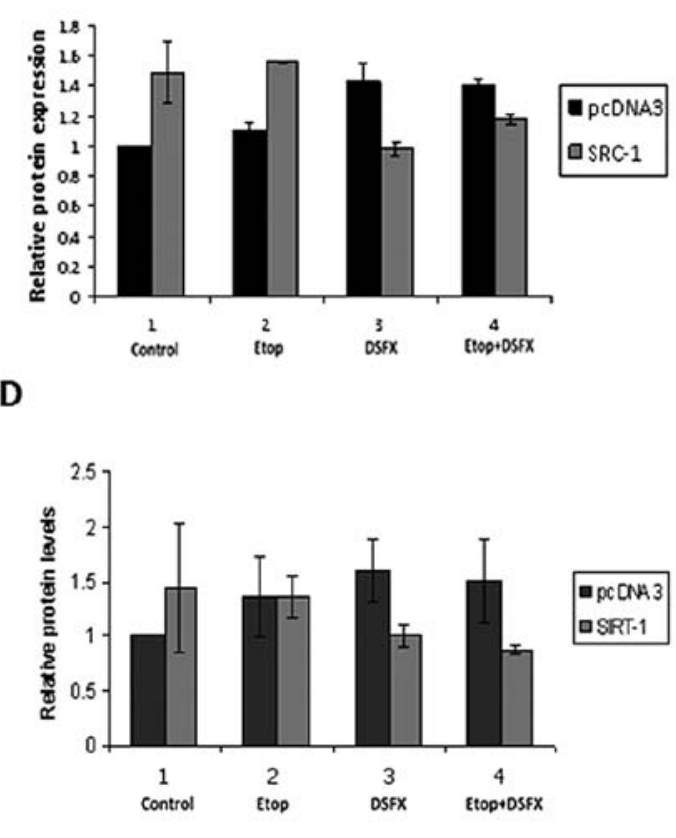

Figure 5. Effect of SRC-1 (A) and SIRT-1 (C) on CXCR4 protein levels in untreated, etoposide, DSFX and both etoposide and DSFX-treated MCF-7 cells. Western blot analysis of HA-SRC-1 (A) Flag-SIRT-1 (C) transfected MCF-7 cells showing the levels of endogenous CXCR4 in unstressed and stressed cells. Endogenous $\beta$-actin levels was used as loading control. Densitometric analysis of 2 independent experiments was performed for A (B) and C (D) using ImageJ software.

fection conferred an $\sim 2$-fold increase in unstressed breast cancer cells compared to pcDNA3 transfected cells (Fig. 5C and D, compare black bar 1 with grey bar 1$)$. However in etoposide, DSFX and etoposide and DSFX-treated cells, SIRT-1 decreased CXCR4 protein levels by 19,34 and $43 \%$, respectively, compared to MCF-7 cells transfected with pcDNA3 (Fig. 5C and D, compare black bars 2-4 to grey bars 2-4, respectively).

\section{Discussion}

Several studies converge to the conclusion that perturbations of the inflammatory response increase the likelihood of pathogenesis in diseases related to metabolism, auto-immunity, and malignant transformation $(5,9,36,37)$. Breast cancer for example is characterized by elevated secretion of pro-inflammatory (IL-1, TNF- $\alpha$, IL-6) or anti-inflammatory cytokines (IL-10), chemokines and chemokine receptors (CXCL8, CXCR4), and angiogenic factors (VEGF) (38). This network of proinflammatory components rivals the inhibitory effect of the anti-inflammatory cytokines (IL-4) on the growth of breast cancer cells (39) and promotes cell proliferation, tumorigenesis and metastasis (9). Thus, it is clear that studying the regulation of the expression levels and activity of cytokines and cytokine receptors is important for understanding the pathways that promote cancer progression.

HIF-1 and NF- $\kappa \mathrm{B}$ act synergistically promoting the expression of pro-inflammatory, pro-angiogenic and pro-survival molecules thus converting pro-inflammatory into oncogenic signals by transactivating gene expression of genes involved in angiogenesis, metastasis and switch of cellular energy metabolism from oxidative phosphorylation to glycolysis (VEGF, GLUT-1, Epo) $(8,17)$. On the other hand, the stress responsive tumor suppressor protein p53 restrains neoplastic transforma- tion via its pro-apoptotic and anti-proliferative function $(20,21)$. In an attempt to provide additional mechanistic insights into the molecular pathways that contribute to breast cancer progression via cancer-related inflammation we evaluated the roles of HIF- $1 \alpha$ and p53 transcription factors in the regulation of the expression of inflammatory cytokines.

In line with earlier studies indicating induction of $C X C R 4$ gene expression in hypoxic breast and ovarian cancer cells (19) elevated CXCR4 mRNA levels were observed in DSFX-treated MCF-7 breast cancer cells compared to those determined in normoxic conditions (Fig. 1A). Furthermore, the inflammatory genes $I L-10$ and $T N F$ - $\alpha$ were upregulated in hypoxia mimicking conditions (Fig. 1B and C). HIF-1 $\alpha$ and NF- $\kappa$ B pathways mediate upregulation of the pro-inflammatory $T N F-\alpha$ levels in hypoxic macrophages (40) but the finding that $T N F-\alpha$ is upregulated in epithelial cancer cells via the same signalling pathways provides an additional indication that inflammation and cancer are closely linked (41).

In an effort to understand the molecular mechanisms involved in $C X C R 4$ and $T N F$ - $\alpha$ gene expression under diverse types of stress, the recruitment of HIF-1 $\alpha$ to the promoter of these genes was followed in MCF-7 cells treated with etoposide or DSFX either individually or in combination. HIF-1 $\alpha$ was recruited to the $C X C R 4$ promoter only in cells treated with combination of etoposide and DSFX whereas in the $T N F-\alpha$ promoter HIF-1 $\alpha$ was detected in the chromatin immunocomplexes of cells treated with both DSFX alone and combination of DSFX with etoposide. Possible explanation for the selective recruitment of HIF-1 $\alpha$ to the $C X C R 4$ or $T N F$ - $\alpha$ promoter could be the different composition of the HIF-1 $\alpha$ transcription complexes in the two cases targeting this transcription factor to one or the other promoter depending on the environmental conditions, or differential HIF-1 $\alpha$ posttranslational modifications. 
To test this hypothesis the contribution of SRC-1 and SIRT-1, which are common modulators of both HIF-1 $\alpha$ and p53 exerting opposing effects on the activity of these transcription factors, to the regulation of $\mathrm{CXCR} 4$ cellular levels was investigated. Our findings suggest that exogenous SRC-1 induced CXCR4 protein levels in normoxic and etoposide-treated MCF-7 cells but repressed its cellular levels in DSFX-treated MCF-7 cells (Fig. 5A and B). Furthermore, exogenously expressed SIRT-1 reduced $\mathrm{CXCR} 4$ protein levels and $C X C R 4$ luciferase reporter activity in DSFX-treated MCF-7 cells (Figs. 5C and D and 4B respectively). Additionally, in MCF-7 cells treated with either etoposide alone or with combination of etoposide and DSFX, SIRT-1 overexpression reduced CXCR4 protein levels compared to non-transfected cells in the same conditions (Fig. 5C and D).

Collectively the data presented here provide evidence that the functional interplay between HIF- $1 \alpha$ and p53 and possibly other transcription factors mediated by SRC-1 and SIRT-1 under diverse types of stress in hypoxic breast cancer cells modulate the gene expression of the inflammatory CXCR4 chemokine receptor.

\section{References}

1. Balkwill $\mathrm{F}$ and Mantovani A: Inflammation and cancer: back to Virchow? Lancet 357: 539-545, 2001.

2. de Visser KE, Eichten A and Coussens LM: Paradoxical roles of the immune system during cancer development. Nat Rev Cancer 6: 24-37, 2006

3. Allavena P, Garlanda C, Borrello MG, Sica A and Mantovani A Pathways connecting inflammation and cancer. Curr Opin Genet Dev 18: 3-10, 2008

4. Lin WW and Karin M: A cytokine-mediated link between innate immunity, inflammation, and cancer. J Clin Invest 117: 1175-1183, 2007.

5. Mantovani A, Allavena P, Sica A and Balkwill F: Cancer-related inflammation. Nature 454: 436-444, 2008.

6. Shiao SL, Ganesan AP, Rugo HS and Coussens LM: Immune microenvironments in solid tumours: new targets for therapy. Genes Dev 25: 2559-2572, 2011.

7. Mantovani A, Sozzani S, Locati M, Allavena P and Sica A: Macrophage polarization: tumor-associated macrophages as a paradigm for polarized M2 mononuclear phagocytes. Trends Immunol 23: 549-555, 2002.

8. Aggarwal BB, Shishodia S, Sandur SK, Pandey MK and Sethi G: Inflammation and cancer: how hot is the link? Biochem Pharmacol 72: 1605-1621, 2006.

9. Balkwill F: TNF-alpha in promotion and progression of cancer. Cancer Metastasis Rev 25: 409-416, 2006.

10. Mocellin S, Marincola FM and Young HA: Interleukin-10 and the immune response against cancer: a counterpoint. J Leukoc Biol 78: 1043-1051, 2005 .

11. Ali S and Lazennec G: Chemokines: novel targets for breast cancer metastasis. Cancer Metastasis Rev 26: 401-420, 2007.

12. Balkwill F: Chemokine biology in cancer. Semin Immunol 15: 49-55, 2003.

13. Allavena P, Germano G, Marchesi Fand Mantovani A: Chemokines in cancer-related inflammation. Exp Cell Res 317: 664-673, 2011.

14. Luker KE and Luker GD: Functions of CXCL12 and CXCR4 in breast cancer. Cancer Lett 238: 30-41, 2006.

15. Balkwill F: The significance of cancer cell expression of the chemokine receptor CXCR4. Semin Cancer Biol 14: 171-179, 2004.

16. Uygur B and Wu WS: SLUG promotes prostate cancer cell migration and invasion via CXCR4/CXCL12 axis. Mol Cancer 10: 139,2011

17. Balkwill F: Cancer and the chemokine network. Nat Rev Cancer 4: 540-550, 2004.
18. Denko NC, Fontana LA, Hudson KM, et al: Investigating hypoxic tumor physiology through gene expression patterns. Oncogene 22: 5907-5914, 2003.

19. Schioppa T, Uranchimeg B, Saccani A, et al: Regulation of the chemokine receptor CXCR4 by hypoxia. J Exp Med 198: 1391-1402, 2003.

20. Mehta SA, Christopherson KW, Bhat-Nakshatri P, et al: Negative regulation of chemokine receptor CXCR4 by tumor suppressor p53 in breast cancer cells: implications of p53 mutation or isoform expression on breast cancer cell invasion. Oncogene 26: 3329-3337, 2007

21. Webster GA and Perkins ND: Transcriptional cross-talk between NF-kappaB and p53. Mol Cell Biol 19: 3485-3495, 1999.

22. Xenaki G, Ontikatze T, Rajendran R, et al: PCAF is an HIF-1alpha cofactor that regulates p53 transcriptional activity in hypoxia. Oncogene 27: 5785-5796, 2008.

23. Blagosklonny MV, An WG, Romanova LY, Trepel J, Fojo T and Neckers L: p53 inhibits hypoxia-inducible factor-stimulated transcription. J Biol Chem 273: 11995-11998, 1998.

24. An WG, Kanekal M, Simon MC, Maltepe E, Blagosklonny MV and Neckers LM: Stabilization of wild-type p53 by hypoxiainducible factor 1alpha. Nature 392: 405-408, 1998.

25. Lee SK, Kim HJ, Kim JW and Lee JW: Steroid receptor coactivator-1 and its family members differentially regulate transactivation by the tumor suppressor protein p53. Mol Endocrinol 13: 1924-1933, 1999.

26. Ruas JL, Poellinger L and Pereira T: Role of CBP in regulating HIF-1-mediated activation of transcription. J Cell Sci 118: 301-311, 2005.

27. Rajendran R, Garva R, Krstic-Demonacos M and Demonacos C: Sirtuins: molecular traffic lights in the crossroad of oxidative stress, chromatin remodeling, and transcription. J Biomed Biotechnol 2011: 368276, 2011.

28. Xu J, Wu R-C and O'Malley BW: Normal and cancer-related functions of the p160 steroid receptor co-activator (SRC) family. Nat Rev Cancer 9: 615-630, 2009.

29. Onate SA, Tsai SY, Tsai MJ and O'Malley BW: Sequence and characterization of a coactivator for the steroid hormone receptor superfamily. Science 270: 1354-1357, 1995.

30. Demonacos $\mathrm{C}$ and La Thangue NB: Drug discovery and the $\mathrm{p} 53$ family. Prog Cell Cycle Res 5: 375-382, 2003.

31. Keimling M and Wiesmuller L: DNA double-strand break repair activities in mammary epithelial cells - influence of endogenous p53 variants. Carcinogenesis 30: 1260-1268, 2009.

32. Carrero P, Okamoto K, Coumailleau P, O'Brien S, Tanaka H and Poellinger L: Redox-regulated recruitment of the transcriptional coactivators CREB-binding protein and SRC-1 to hypoxiainducible factor 1alpha. Mol Cell Biol 20: 402-415, 2000.

33. Lavery DN and Bevan CL: Androgen receptor signalling in prostate cancer: the functional consequences of acetylation. $J$ Biomed Biotechnol 2011: 862125, 2011.

34. Zhang Q, Tang X, Lu QY, Zhang ZF, Brown J and Le AD: Resveratrol inhibits hypoxia-induced accumulation of hypoxiainducible factor-1alpha and VEGF expression in human tongue squamous cell carcinoma and hepatoma cells. Mol Cancer Ther 4: 1465-1474, 2005.

35. Lim J-H, Lee Y-M, Chun Y-S, Chen J, Kim J-E and Park J-W: Sirtuin 1 modulates cellular responses to hypoxia by deacetylating hypoxia-inducible factor $1 \alpha$. Mol Cell 38: 864-878, 2010.

36. De Nardo D and Latz E: NLRP3 inflammasomes link inflammation and metabolic disease. Trends Immunol 32: 373-379, 2011.

37. Strober W and Fuss IJ: Proinflammatory cytokines in the pathogenesis of inflammatory bowel diseases. Gastroenterology 140: 1756-1767, 2011

38. Mantovani A, Marchesi F, Porta C, Sica A and Allavena P: Inflammation and cancer: breast cancer as a prototype. Breast 16 (Suppl 2): S27-S33, 2007.

39. Toi M, Bicknell R and Harris AL: Inhibition of colon and breast carcinoma cell growth by interleukin-4. Cancer Res 52: 275-279, 1992.

40. Murdoch C and Lewis CE: Macrophage migration and gene expression in response to tumor hypoxia. Int J Cancer 117: 701-708, 2005.

41. Hussain SP and Harris CC: Inflammation and cancer: an ancient link with novel potentials. Int J Cancer 121: 2373-2380, 2007. 